\title{
ALCHIMIA, PRELUDIO ALLA CHIMICA?
}

\author{
Nota del m.e. GIANNANTONIO SACCHI LANDRIANI (*)
}

(Adunanza del 21 aprile 2016)

SunTO. - Scopo di questa comunicazione è la ricerca, nella Biblioteca scientifica antiquaria dell'Istituto Lombardo, di documenti che preludano alla chimica. Come noto la chimica ha avuto un rigoroso assetto alla fine del XVIII secolo. Di fatto abbiamo trovato alcuni libri concernenti complicate e raffinate empiriche tecnologie particolarmente relative alla lavorazione di metalli e alla realizzazione di colori per la realizzazione di ceramiche. È necessario ricordare che durante la Rinascenza il clima culturale scientifico era alchimistico. La diffusione di informazioni riguardanti queste attività erano gelosamente custodite fra i membri delle corporazioni. Questa può essere considerata una ragione della povertà di documenti originali.

$* * *$

ABSTRACT. - This paper aims to a review, in the antiquarian scientific library of the Istituto Lombardo, able to find forerunner documents of chemistry. As it is well known chemistry found a rigorous settlement at the end of XVIII century. Actually we have found some books concerning complicated refined empirical technology particularly devoted to metal and making of colours for ceramic processing. It is necessary remember that during Renaissance the scientific cultural climate was alchemistical. So the spread of information concerning this kind of activities among members of guilds was nearly secret. This is the reason why the number of documents found is very poor.

\section{CONSIDERAZIONI INTRODUTTIVE}

Il proposito di questo intervento è dettato dal desiderio di avvicinarci alla conclusione di un itinerario iniziato da un paio d'anni. In precedenti comunicazioni avevamo presentato notizie relative a esplorazioni di cinquecentine scientifiche, appartenenti alla biblioteca del nostro

(*) Politecnico di Milano, Istituto Lombardo Accademia di Scienze e Lettere, Milano, Italia. E-mail: glansacchi.landriani@gmail.com 
Istituto, in tema di matematica e fisica, di medicina e scienze naturali, di astronomia. Sembrava non irragionevole (al fine di ampliare l'esplorazione dei documenti scientifici del XVI secolo) rivolgere l'attenzione a quanto poteva preludere alla nascita della chimica, coscienti della circostanza che questa disciplina avrebbe avuto un assetto scientificamente definito a partite dal XVIII secolo.

Si tratta di un patrimonio librario (quello strettamente relativo a questo argomento) dotato di un numero esiguo di esemplari (e modesto contributo è pubblicato in cataloghi generali, quali ICCU e EDIT16).

Gli ingegnosi pensieri e le drammatiche speranze del XVI secolo erano, sotto la cappa dell'Astrologia, un insieme di tentativi di osservazioni di fenomeni complessi, di conclusioni fumose, ma formulate con acume, fantasia e ingegno. In quanto segue daremo rilievo ad alcuni autori che ci sembrano i più significativi, tra quelli della nostra biblioteca, quali anticipatori di concetti propri di innovatrici tecnologie.

La circostanza che nel XVI secolo la cultura non fosse organizzata, come l'attuale, in distinte discipline definite in ambiti precisi, rende complesso il discernere in un autore quanto potesse preludere alla non ancor definita Chimica.

Tutto era confluente in un ambito immaginifico dove la botanica, l'astronomia, la farmacologia, la medicina, confinavano con la teologia o con la sapienza aristotelica.

E' ammirevole comunque la profonda aspirazione degli antichi autori a prefigurare una conoscenza organizzata.

Significative notizie sono contenute nell'ambito di dottrine dalla forte tradizione come la botanica, la farmacia, l'anatomia.

Un argomento che merita una particolare attenzione è relativo alla preparazione dei colori (grandi vetrate di cattedrali, dipinti, affreschi).

Il Cinquecento è il secolo d'oro della maiolica italiana. Richiesta dai Signori, le stoviglie istoriate ormai di svariate forme, facevano bella mostra nei saloni ed erano spesso affiancate da stoffe pregiate e suppellettili in oro e argento, quali simboli di potenza economica.

La produzione ceramica in questo periodo fece un salto di qualità, arrivando a dialogare strettamente con la pittura contemporanea (spesso copiandone le composizioni grazie al circolare delle incisioni) con la nascita di botteghe di pittori specializzati in questo tipo di decorazioni.

Le tecniche utilizzate presupponevano conoscenze empiriche complesse relative al valore cromatico di una sostanza, tenendo conto delle dimensioni degli oggetti e delle particelle usate nei pigmenti. La 
composizione dei colori era eseguita secondo ricette spesso tenute segrete dagli artefici, o nell'ambito delle specifiche corporazioni.

La composizione chimica dei materiali usati in quell'epoca è stata, e non completamente, analizzata soltanto a partire dal XIX secolo. Oggi lo è sulla base delle proprietà studiate per sistemi nanotecnologici.

Si ha notizia di interessanti contributi cinquecenteschi, che citano la problematica connessa alla produzione di colorazioni e pertanto con osservazioni che appartengono a prolegomeni della futura chimica, almeno dal punto di vista tecnologico.

Stupisce che Giorgio Vasari (1511-1574) ne LE VITE, opera edita nel 1550, ammette di non aver saputo (o forse voluto) svelare i segreti degli smalti, dei colori, delle arti applicate alla tecnologia del fuoco (vetro, mosaico, ceramica).

Grande notorietà ebbe all'epoca, e tutt'ora la conserva, l'opera del 1548 di Cipriano Piccolpasso (1524-1579) [riprodotta per intero alla pagina line Google] LI TRE LIBRI DELL'ARTE DEL VASAIO [IN QUIETEIntroduzione di Giulio Vada MMII] il cui contenuto è dedicato in gran parte alla smaltatura delle terrecotte con interessanti indicazioni, non certe ma attendibili, sugli elementi e relative applicazioni usate da Luca della Robbia nel XV secolo e dai suoi eredi nel XVI e XVII secolo.

Si tratta di un'opera che tende a svelare i segreti dei «vasari» senza tuttavia abbordare una elaborazione abbastanza sistematica da appoggiare ad un tecnica scientifica. Non è trascurabile che il Piccolpasso fosse coevo ed interlocutore di Donatello e di Brunelleschi. Inoltre è opportuno ricordare che la ceramica arrivò a dialogare con i pittori contemporanei e con le loro botteghe.

Il procedimento contemplava la preparazione e la foggiatura dell'impasto argilloso, la sua essicazione e cottura a biscotto, quindi la smaltatura. Materia prima è sabbia, silice non inquinata da ferro o titanio.

La silice fonde a più di $1700^{\circ} \mathrm{C}$, temperatura non raggiungibile allora, ed era quindi necessario aggiungere fondenti per aumentarne la fusibilità e però assicurare la scorrevolezza degli smalti ossia alcali, sodio, potassio, e piombo. Era importante fondere il piombo con la silice (silicato di piombo) ossia vetro a bassa fusibilità dotato di alta brillantezza (che si deve al piombo). 3 parti di piombo+1 parte di sabbia.

Il Piccolpasso afferma di conoscere il segreto di spalmare l'ORPIMENTO (pigmento d'oro+solfuro d'arsenico in cristalli di color giallo oro) sui bordi del BISCOTTO (terracotta o ceramica di prima cottura destinata a ricevere il rivestimento vetroso). 
Un adagio del tempo affermava:

L'ossido di stagno conferisce bianco. L'arsenico dà brillantezza.

Il piombo fa lustro, lo stagno fa bianco.

La stima della temperatura era effettuata, in quel tempo precedente all'invenzione dei termometri, mediante l'osservazione ad occhio nudo del colore assunto dal pezzo in lavorazione.

Le tecniche utilizzate presupponevano conoscenze empiriche complesse relative a colori, dimensioni, particelle usate nei pigmenti.

Benché non strettamente attinente al tema di prologo della chimica, ma piuttosto alla fisica (in particolare l'ottica) e con accenni di anatomia (oftalmia), comunque con caratteristiche scientifiche marcate e preveggenti per l'epoca, è l'opera, presente nella nostra biblioteca di Cartesio [RENATUS DES CARTES] (1569-1650) dal titolo de HOMINI FIGURIS. In buono stato di conservazione consta di 122 pagine + tavole fuori testo, h $20 \mathrm{~cm}$.

\section{L'oPeRA DEL SENESE VANNUCCIO BIRINGUCCIO}

Al senese Vannuccio BIRINGUCCIO (1480-1539), autore significativo nella storia della scienza, ma soprattutto della tecnologia, dobbiamo riconoscere notevole maestria nella presentazione di tecniche applicate alla produzione di oggetti metallici, come illustreremo nel seguito. Tecnologie che facevano appello all'uso della fusione dei metalli per creare armi, oppure campane, tanto che troviamo nel frontespizio della sua maggiore opera la sintesi del contenuto che segue.

P I R O T E C H N I A

L I D I E C I L I B R I D E L L A

P I R O T E C H N I A

Nelli quali fi tratta non solo la diuerfita

delle minere, ma ancho quanto fi ricer

ca alla prattica di effe:e di quan/l

s'appartiene all'arte della

fufione ouer getto de me

talli,e d'ogni altra co/l

fa a quefta fo/l

migliante

Far campane, artiglieria, fuochi artificiali e diuerse cose utilissime. 
Per ottenere pregevoli risultati ci si valse della sapienza ereditata da antichi studiosi esperti nell'individuare le miniere dalle quali trarre il grezzo per isolare soprattutto metalli. E con questi creare leghe quali il bronzo, l'ottone, l'acciaio.

Nella stessa utopia alchimistica, di trasformare qualunque vile sostanza in oro, v'è un germe delle trasformazioni tipiche dell'attuale disciplina chimica.

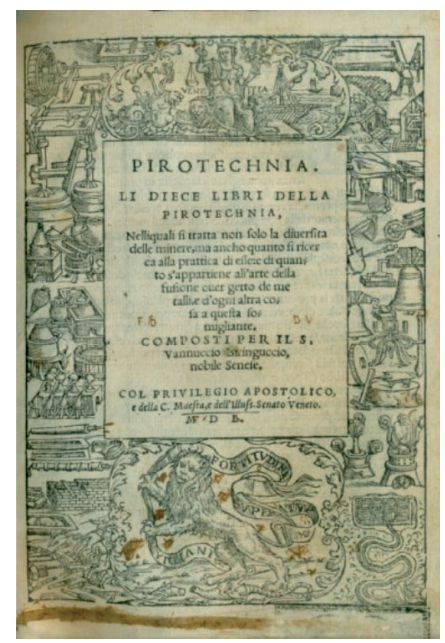

Fig. 1 - L A PIROTECHNIA LI DIECI LIBRI DELLA PIROTECHNIA edizione 1550.

In Fig. 1 si presenta L A PIROTECHNIA LI DIECI LIBRI DELLA PIROTECHNIA editi per il s. Vannuccio Biringuccio nobile senese (1480-1539) COL PRIVILEGIO APOSTOLICO, e della C.Maestà dell'Illuss. Senato Veneto nel MDL

Si tratta probabilmente di una seconda edizione. Questa edizione del MDL non è catalogata ICCU.

La prima edizione citata [IT/ICCU/RAV/1930982] sembra essere del 1540 con un solo esemplare. Ma gode di ampia bibliografia del XX secolo.

In Fig. 2 è presentata l'edizione datata MDLIX in VENETIA Appresso Geronimo Giglio e compagni. Si tratta di un esemplare purtroppo fortemente danneggiato in frontespizio e con scritte a mano

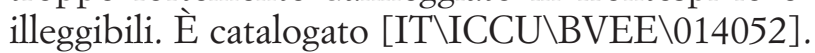

Si tratta di una pubblicazione avvenuta un decennio dopo la 
morte dell'autore. Molto diffusa all'epoca e tuttora molto studiata dagli specialisti.

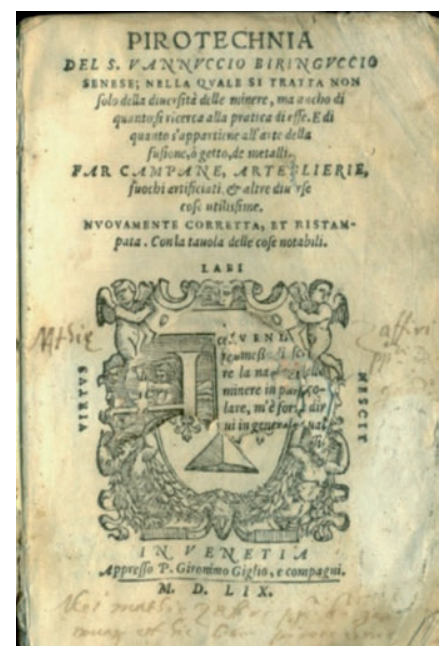

Fig. 2 - Successiva edizione datata MDLIX.

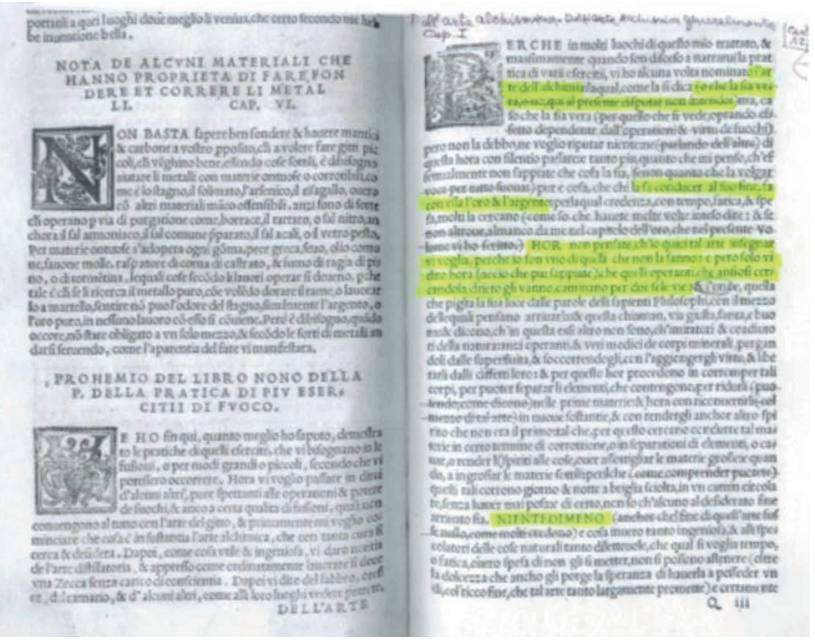

Fig. 3 - La pagina 122 dedicata al PROEMIO DEL LIBRO NONO.

In Fig. 3 è rappresentata la pagina 122, dell'edizione del MDL, dedicata al PROEMIO DEL LIBRO NONO con DELLA PRATICA DI PIÙ ESERCIZI DI FUOCO (cartella 123). 

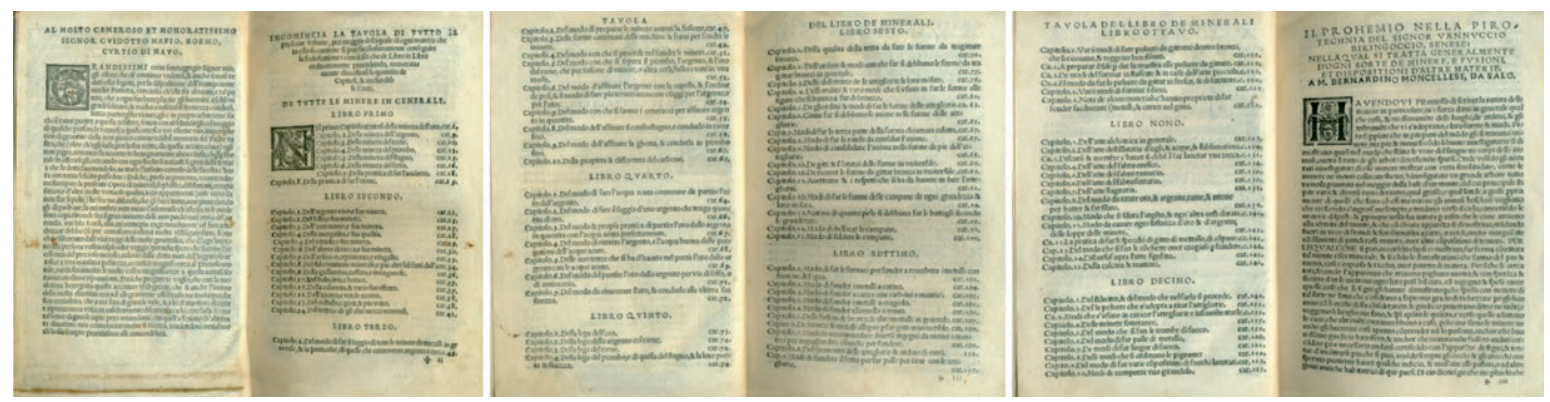

Fig. 4 - Completo indice analitico di PIROTECHNIA edizione del MDL.

L'insieme della Fig. 4 è la presentazione completa dell'indice analitico di PIROTECHNIA edizione del MDL. I dieci libri sono descritti con precisione e trattano delle miniere d'argento, rame, piombo, ferro e della pratica di fare l'acciaio e l'ottone. Indicano inoltre come lavorare i minerali per isolare gli elementi con tecniche di fonderia.

\section{CONSIDERAZIONI DI BIRINGUCCIO SULL'ARTE ALCHIMICA}

La pagina a destra (Fig. 3) è dedicata al tema DELL'ARTE ALCHIMICA IN GENERALE cap I (car 123).

Sembra opportuno tracciare una sintesi delle considerazioni in tema di Alchimia di Biringuccio, riconoscendogli una notevole capacità di preveggenza sugli sviluppi della conoscenza.

L'autore, nelle cartelle 123 e 124 della sua opera, si chiede cosa sia "l'arte alchimica che oggi con tanta cura si cerca e desidera".

Premette la distinzione di due vie di praticare l'alchimia: la prima rispettabile ancorché immatura, la seconda cattiva ed esecrabile. Si pone in una posizione prossima alla prima pur dimostrando eccezionale capacità critica.

In particolare riconosce la notorietà della prima via pur essendo scettico sui risultati, consistenti essenzialmente nella trasformazione della materia con il miraggio di ottenere oro e argento. Ma è un estimatore di quanti, da lui definiti buoni alchimisti, cercano di capire in definitiva la natura delle materie, in particolare metalliche. Si professano medici dei corpi minerali e collaboratori della natura in quanto cercano di purgare i detti corpi dalle impurità, di liberarli dei difetti e se necessario di aggiungere virtù. La tecnica ricorrente è quella della frantuma- 
zione, del ridurre in polvere per isolare le sostanze di base, e mescolandole di trovare nuove sostanze.

In generale costoro soni colti, rigorosi e in itinere cercano di trovare benefici per l'umanità. Esempio fondamentale è lo sviluppo della farmacopea. Il buon alchimista non deve essere povero, poiché la sua disciplina è costosa; non deve essere avaro, perché moralmente tenuto a far conoscere i propri risultati; deve essere un genuino amante della conoscenza.

Biringuccio ha parole severe nei confronti di coloro, definiti cattivi alchimisti, che si valgono dell'alchimia con mala intenzione. Operano con tecniche scorrette e disordinate (per esempio nella cattiva gestione dei fuochi) e vogliono spacciare l'ottone come oro, il vetro come cristallo, gli smalti come gemme. Si comportano in sostanza come avidi imbroglioni.

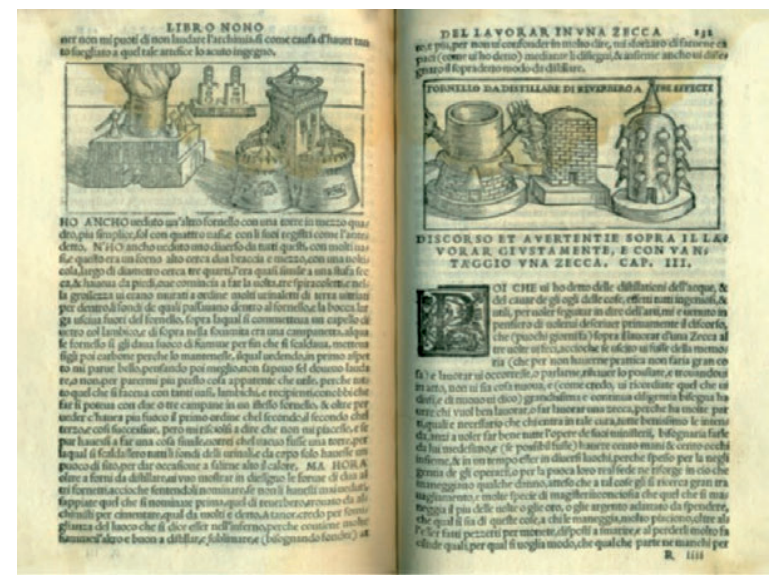

Fig. 5 - Forni per la distillazione con molti alambicchi.

Nella Fig. 5 vengono illustrati accuratamente forni per la distillazione con gli scarichi dei fumi e soprattutto dotati di molti alambicchi atti a raccogliere all'esterno il distillato.

Si noti nella pagina di destra l'illustrazione di forni a riverbero. L'autore desidera mostrare in disegno le forme di due altri fornetti. Il primo, quello a riverbero, "inventato" da alchimisti e realizzato per contenere molte fiamme. Il secondo permette di distillare, sublimare, e fondere. Ingegnosa e delicata la tecnica del forno a riverbero perché il calore è fornito da un gas ad alta temperatura che lambisce il materiale 
in trattamento. Una difficoltà rilevante è connessa con la circostanza che all'epoca le temperature facilmente ottenibili si aggiravano sui novecento gradi centigradi (con stima a vista) generati per lo più da polvere di carbone combusta.

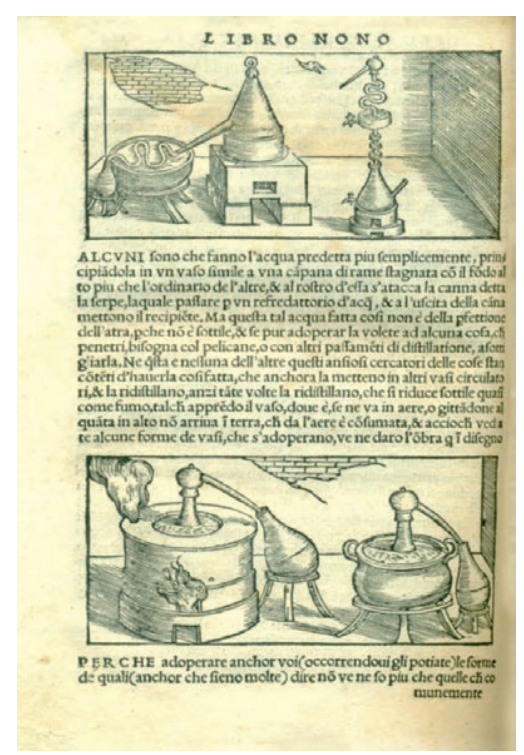

Fig. 6 - Tecniche di raffreddamento del distillato.

Nella Fig. 6 si illustrano altri forni per la distillazione con particolare riguardo alle tecniche di raffreddamento del distillato prima di essere raccolto nei vasi. Si illustrano serpentine per prolungare il percorso del distillato, e tecniche di stabilizzazione della temperatura di distillazione con dispositivi ad acqua in Bagno-Maria.

In Fig. 7 è illustrata una raffinata tecnica per "far l'oro in filo". Si illustra la possibilità di realizzare sottili cavi la cui anima sia d'argento o rame e soltanto la parte esterna in oro. Il fine è di ridurre il costo di realizzazione sí che risplendano come auree massicce (come ad esempio nel caso di uniformi militari).

Si realizza facilmente una verga sottile d'argento o di rame. La si lavora a martello e la si rifinisce a lima. Si introduce poi in un fornello con carbone e fiamma d'ontano, portandola sino a quasi fusione per poterla infine trafilare sí da renderla "TANTO FINE CHE QUASI L'OCCHIO NON COGLIE”. 


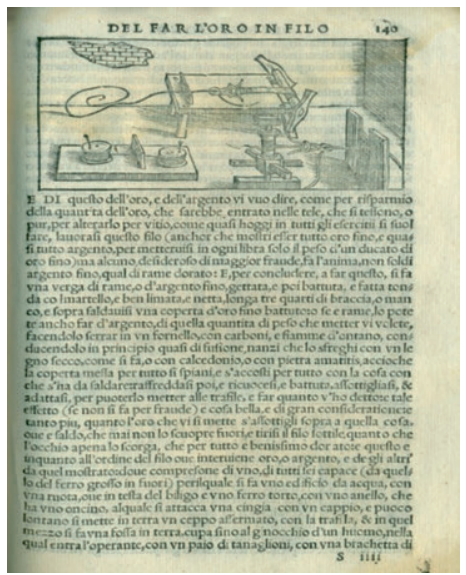

Fig. 7 - Dispositivo per produrre fili d'oro a partire dal massello.

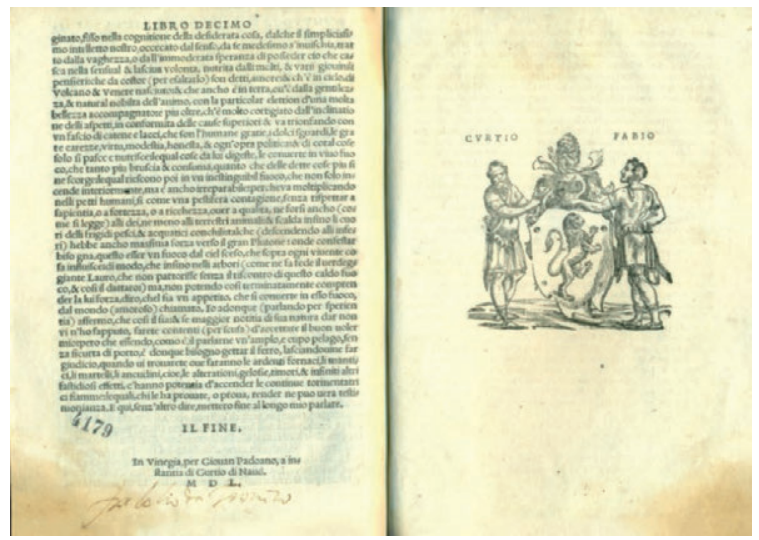

Fig. 8 - L'ultima pagina dell'opera di Vannuccio Biringuccio.

Nella pagina (Fig. 8) di chiusura la marca è di estrema raffinatezza grafica con l'indicazione del nome dell'editore CURTIO FABIO. Si noti che la dicitura al piede della pagina precedente recita: IL FINE e la dicitura in Vinegia, per Giouan Padoano, a istantia di Curtio Nauò. Segue una scritta a mano, probabilmente un EX LIBRIS. $\mathrm{h} \mathrm{cm} 21$, pag. 169.

Molto chiara la data di edizione MDL. Segue una firma difficilmente interpretabile. 


\section{DUE OPERE DI IMPOSTAZIONE ALCHIMICA}

Nelle due Figure seguenti (Figg. 9-10) si illustrano due opere non propriamente relative ad aspetti riguardabili come prolegomeni della chimica, ma piuttosto da ritenersi contenitori di aspetti molto elementari della chimica. Sono infatti destinati allo studio della farmacopea e costituiscono comunque due reperti interessanti dal punto di vista bibliografico orientato alla scienza.

In Fig. 9 è presentata un'opera dal titolo Examen omnium Syruporum publicus vfus [usus] est.

Si tratta di un volume contenente tre contributi di carattere terapeutico. I primi due sono dello stesso autore indicato in figura dedicati a medicamenti. Il primo, di pagine 131+index, è edito in VENEZIA MDXLV e il secondo, di pagine 205, è datato MDXLIX. In [ICCU $\backslash B V E E \backslash 0011476]$ si dichiara l'esistenza di 17 esemplari. Preziosa la marca dell'editore.

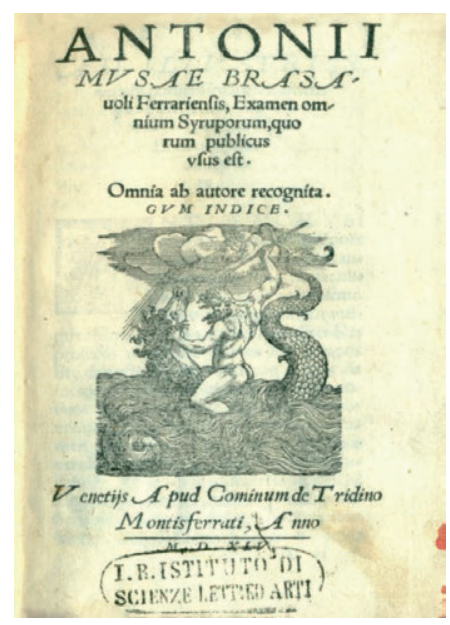

Fig. 9 - Volume contenente due contributi di ANTONIO MUSA BRASAVOLA ferrarese (1500-1555) ed un contributo di ORIBASIO SARDANI.

Il terzo contributo è di ORIBASIO SARDIANI ed è legato nel volume precedente. Dedicato alla preparazione di medicinali. Edito in VENETIJS MDLVIII reca un ex libris scritto a mano che potrebbe leggersi Ex Zacharia' Caimi, h cm 14,7, pagine 216+note+index.

L'intera opera contiene tracce di attenzione a elementi di studio 
analitico che potrebbero indurre ad un vago preludio di sistematica chimica. Si tratta di opera in buono stato di conservazione e di un certo pregio bibliografico.

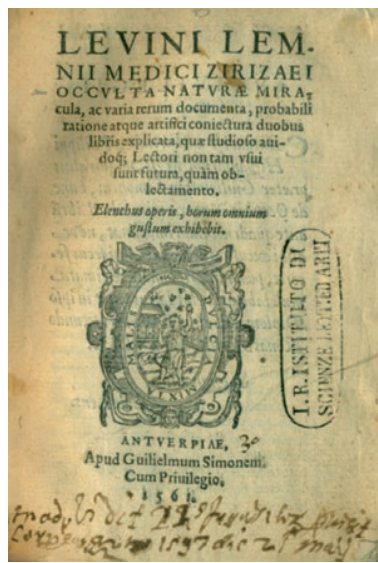

Fig. 10 - Opera di LEVINO LEMNO (1505-1568).

In Fig. 10 è presentata l'opera di LEVINO DEMNIO dal titolo Occulta Natura Miracula, pag. 164, in folio h cm 14,5. Rilegatura originale, stato di conservazione mediocre, prima pagina interamente strappata, molte scritte a mano in inchiostro quasi illeggibili, timbro IL del periodo Lombardo-Veneto. In basso alla pagina di frontespizio una scrittura leggibile nella parte terminale con la dicitura 1597 die 21 may, nella seconda pagina di copertina una lunga scritta di difficile interpretazione. Presenta un elenco numerato degli argomenti trattati, seguito da RERUM ET SENTENTIARUM HUIUS OPERIS INDEX, tratta di malattie fisiche e psichiche senza indicazioni terapeutiche e con un linguaggio ispirato ad interpretazioni misteriose e magiche.

Quest'opera ha avuto una grande fortuna editoriale come risulta dalla lista che segue:

ANTWERPIAE 1561 ed Guillelmum Simonem, in latino, non catalogata ICCU, VENETIA 1560 ed Ludovico Auanzi, in italiano, 14 esemplari in [ICCU $\backslash B V E E \backslash 004751]$

1563 idem IT 16 esemplari in [ICCU $\backslash B V E E \backslash 004802$ ]

1567 idem IT 12 esemplari in [ICCU $\backslash B V E E \backslash 004821]$

1573 COLONIAE ed Johann Birkmann, in latino, 2 esemplari in IT[ICCU\BVEE $\backslash 004825]$ 


\section{Un'opera POSTUMA Di PARACELSO}

Dotato di una personalità prepotente TEOPHRASTUS PARACELSO (Einsiendeln 1493- Salisburgo 1541) si è dedicato a vastissimi ambiti culturali. Si professa infatti medico, con qualche lampo di fisiologia, farmacologo nuovissimo perché fu tra i primi ad affermare che anche sostanze minerali, e non soltanto vegetali come sino ad allora si riteneva, potessero proficuamente essere utilizzate per creare medicamenti. Non disdegna di valersi s'una vaga psicologia che appartiene disciplinarmente alla magia, definisce lo zolfo come nuovo elemento. Un aspetto di rilevante interesse risiede nei commenti finali agli Aforismi di Ippocrate. Gli Aforismi non hanno specifico carattere riguardante la chimica, ma interessanti osservazioni riguardanti l'atteggiamento corretto di chi intende dedicarsi a studi scientifici.

Embrioni di chimica vanno cercati piuttosto nei ricettari e negli elenchi di elementi come vedremo illustrati.

Fu coevo, all'università di Ferrara, di Copernico. Fu corrispondente di Marsilio Ficino e Piero della Mirandola.

La concezione che Paracelso ebbe della scienza tradisce una notevole apologia.

Riteneva la scienza fosse "talmente alta" da non poter essere raggiunta con lo studio, come comunemente veniva sostenuto, ma d'essere accessibile solo per rivelazione alla quale si poteva a sua volta giungere con la perfetta conoscenza di Dio. Lo studio doveva quindi essere inteso come un mezzo soltanto necessario per raggiungere la scienza.

Alcuni autori hanno attribuito a Paracelso un concetto globale di Scienza e Religione.

A lui viene attribuita l'espressione:

Come si conosce la Scienza nella conoscenza di Dio, così si giunge meglio a Dio nella conoscenza della Scienza.

Paracelso avversava Aristotele perché ne rifiutava i quattro fondamentali elementi (aria, terra, acqua, fuoco). Nel linguaggio di Paracelso sono presenti elementi squisitamente astrologici e aspirazioni alla futura scienza in particolare chimica. Singolare la pretesa di spiegare una fenomenologia cosmica con termini chimici: certi astri sulforeggiano, altri arsenicano, altri salano, altri mercuriano. Nel mare magnum cioè 
nell'atmosfera in tal modo procurano le malattie. Sottolineare la necessità di fornire all'organismo i minerali (che sono nel macrocosmo e deficienti nel microcosmo).

Il vero scopo della chimica non consiste nella preparazione dell'oro, bensi nella preparazione della medicina (Iatrochimica).

In tempi d'assenza di definiti metodi sperimentali, Paracelso predicava la necessità del lavoro di laboratorio e di osservazione, soprattutto in medicina.

Col nome di chimico indicava chi preparava medicine, estratti e sali. Chi eseguiva ricerca chimica veniva definito "philosophus per ignem".

Una interessante raccolta di Afforismi di Ippocrate commentati da Paracelso si trova nell'Opera Theatro d'Arcani di Ludovico Locatelli edita a Milano (Fig. 11) nel M:DC:XLIV, stato di conservazione mediocre, pag. 436, molte scritte a penna indecifrabili, contributo di Paracelso da pag. 374, Copertina originale, h. cm 17.2.

In Fig. 12 si presenta la pagina 174 iniziale del testo dovuto a Paracelso. Segue nella Fig. 13 la lista intitolata Esplicazione de nomi de Filosofi dedicata a nomenclatura di sostanze dovute alle nomenclature dei sapienti, ovvero filosofi, e seguita dalla dicitura volgare. Si rilevi la preoccupazione di divulgare la conoscenza "scientifica" atteggiamento proiettato sul futuro di un autore, come Paracelso decisamente Alchimista.

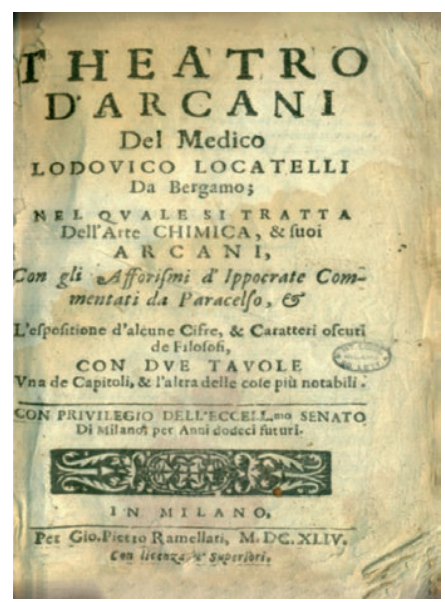

Fig. 11 - Frontespizio del Teatro d'Arcani. 


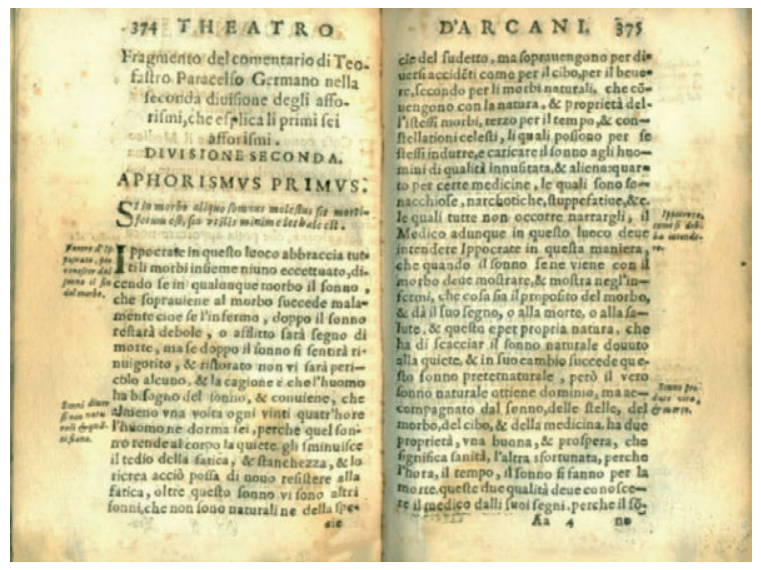

Fig. 12 - Inizio del testo di Paracelso.

Nei commenti di Paracelso appare almeno in due circostanze l'espressione CHIMICA.

Infatti, con riferimento al TEATRO D'ARCANI, si legge:

alla riga 20 di pagina 402, Perché tù possi intendere li libri CHIMICI, che ti capiteranno nelle mani ...

alla riga 9 di pag 403, ... vocaboli of curi che , chi non è buon CHIMICO vero figlio dell'arte non è possibile intenderli...

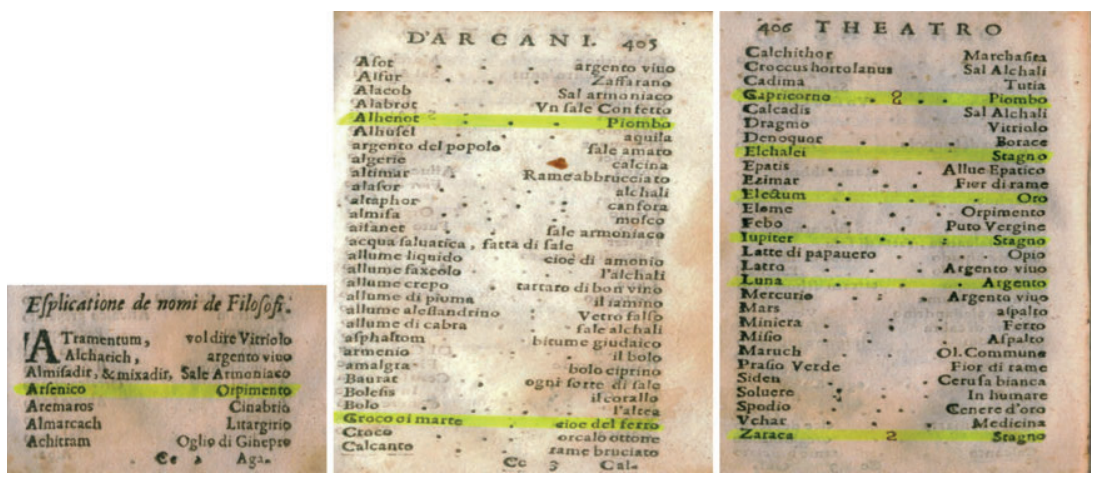

Fig. 13 - Lista dei nomi dati dai Filosofi e usati nel volgare.

Si tratta di citazioni esplicite della parola CHIMICA per intendere fenomeni di difficile interpretazione alla luce dell'alchimia. 


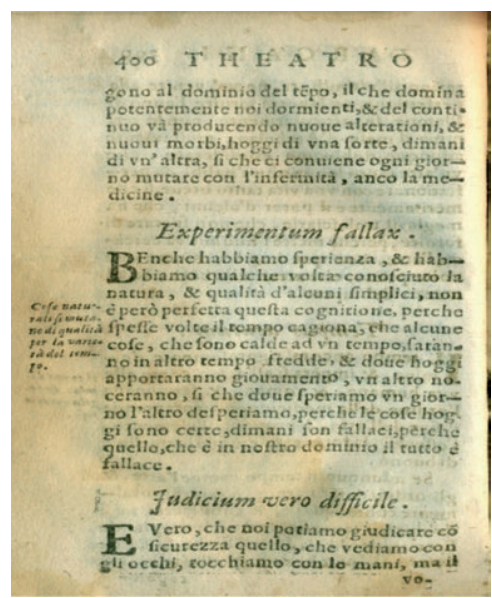

Fig. 14 - Chiusa dei commenti di Paracelso agli AFFORISMI.

Nella Fig. 14 è rappresentata una pagina della chiusa dei commenti di Paracelso agli aforismi di Ippocrate con i paragrafi: Experimentum fallax e Judicium vero difficile. Si presenta una sintesi dei due contributi la cui impostazione epistemologica sembra essere di sorprendente attualità. Il che risulta particolarmente interessante qualora si pensi che si debbono ad un autore la cui attività ha avuto luogo nella prima metà del XVI secolo.

Experimentum fallax

Le cose naturali mutano di qualità con lo scorrere del tempo. ... le cose che oggi sono certe domani saranno fallaci, perché quanto è nel nostro dominio è tutto fallace.

Judicium vero difficile

E' vero che possiamo giudicare con sicurezza quello che vediamo con gli occhi e tocchiamo con le mani. Ma il voler giudicare quello che l'uomo immagina non è cosa facile, perché mentre giudichiamo inganniamo noi stessi con un'opinione, e formuliamo giudizi di quanto non abbiamo mai visto né conosciuto avendo investigato con argomenti sofistici e fallaci ... [Noi] vediamo con gli occhi solamente il corpo, ma non possiamo vedere lo spirito nel quale è posto il fondamento. 


\section{OPERE (XVI-XVII sec.) CONSULTATE}

BIRINGUCCIO Vannuccio, (1550) Pirotechnica, Venezia MDL. BIRINGUCCIO Vannuccio (1559) Pirotechnica, Venezia MDLIX.

LOCATELLI Lodovico (1644) Teatro d'Arcani, Milano MDCXLIV.

DES CARTES Renatus (1662) De Homine Figuris, MDCLXII s.i. h 20.4 cm.

GESNERUM (TEGURINUM) Conradum (s.d.) Historia plantarum (a penna, pag. $281+$ contributo di Theofrasto) h $14,5 \mathrm{~cm}$. .

LEVINI LEMNII (1561)... Occulta naturae miracula... Testo latino, Anrverpiae, h $24,3 \mathrm{~cm}$.

MUSAE BRASA'UOLI Antonii (1545) SCIROPPI,... Venezia MDXLV, h 14,7 cm.

PARACELSO THEOPHRASTI, COMMENTI ad Aforismi di Ippocrate (in THEATRO D'ARCANI di Locatelli Lodovico). Milano MDCXLIV.

PICCOLPASSO Cipriano (1548) Li Tre Libri dell'arte Del Vasaio, Firenze MDXLVIII. 
